\section{MAXIMISING YOUR BRAND}

Keeping a close eye on income and expenditure remains a top priority for practices still coping with the effects of the recession and the requirements of compliance. Under these conditions practitioners are increasingly seeking value for money from their suppliers, as well as innovative ways of encouraging routine attendance by patients.

Dental payment plans provide the answer for patients who want to budget for their regular oral care needs and at the same time ensures predictability for the practice. Once a plan has been launched, practices can look forward to a stable income even during holidays or periods of natural slowdown, so when other practices are suffering from cancellations or absence, those with payment plans continue to receive a steady monthly income.

Making sure you don't waste money on unnecessary additional services makes financial sense and DPAS is still the lowest cost of the three major providers of dental plans. DPAS offer all the additional support necessary to launch and run your payment plan and a partnership can really benefit your practice financially.

Whether you're planning a move into private practice, or already have plans in place, DPAS can help you maximise the value of your practice brand and enable you to benefit from improved cash-flow and stable revenue streams. Reader response number 60

\section{FIGHTING BREATH MALODOUR}

The new Pro-Expert toothpaste from Oral-B is remarkable not only in delivering protection against gum problems, plaque, caries, calculus formation, erosion and dentinal hypersensitivity, but also because it has been shown to reduce oral malodour due to its antibacterial activity and capture of breath malodour molecules.

Breath malodour results from volatile sulphur compounds (VSCs) produced by certain bacteria particularly residing on the tongue. Oral-B ProExpert toothpaste uses the stannous

\title{
COMPLIANT, BEAUTIFUL INTERIORS
}

Dental Design and Planning

Consultants, or DDPC, celebrate 40 years in business with the launch of a new name, 'Designclinic' and website. The company's ethos hasn't changed it continues to deliver compliant and beautiful interiors. Its core business remains the same - providing architecture and interior design services to the dental profession.

Whether you are NHS or private, the Designclinic team have a clear understanding of what makes a practice appealing, profitable, and relaxing. They are capable of undertaking every element of best practice design, taking the headache away from dealing with planners, suppliers, builders and contractors to deliver a project on time and within budget.

They are renowned for offering a personal, discreet and unique service to all their clients. Their standards are exacting and their attention to detail is unrivalled within the dental industry. It is this level of service which makes their projects feel as close to perfection as possible, giving them international recognition. Their new website also showcases recent projects in Moscow and Munich.

Reader response number 61

\section{A WORKHORSE BUILT TO LAST}

New to Bien-Air's innovative range is the Bora Blackline turbine. Thanks to the carbon fibre handle these incredibly light handpieces offer improved resistance to wear, friction, torsion and impacts. The light weight of these handpieces makes them exceptionally easy to manoeuvre with significantly less hand fatigue for the practitioner. LED lighting is standard with all Blackline turbines offering unsurpassed visibility in the operative field.

The Bora is an incredibly quiet turbine from Bien-Air, due to the ceramic ball bearings. Already known as a workhorse, the Bora is built to last.

Bien-Air Bora Blackline and

Metaline turbines are fitted with an integral non-return valve to help with infection control and large push buttons for easy bur removal. These turbines incorporate an anti-heat system to avoid heat build-up during treatment which may cause patient discomfort. The triple spray diffuser with three separate air and water ports ensures optimum cooling even at high speeds. The Unifix quick-connect coupling means that no time is wasted when changing your handpieces.

Reader response number 62 fluoride in its unique combination of polyphosphate and stabilised stannous fluoride to fight the bacteria that produce VSCs by both eliminating them and inhibiting their metabolism. Stannous fluoride complex can also capture the VSCs produced by the bacteria and make them odourless.

This dual approach ensures that the bacteria and their adverse effects in the mouth are reduced, helping to prevent the causes of oral malodour.

By inhibiting plaque growth and bacterial adhesion, stannous fluoride helps prevent the build-up of microbes that are known to exacerbate oral malodour by accumulating in periodontally affected pockets as well as multiplying on the tongue.

The overall benefits of good oral health promoted by use of new Oral-B ProExpert toothpaste are further enhanced by fresh breath and a confident smile. Reader response number 63

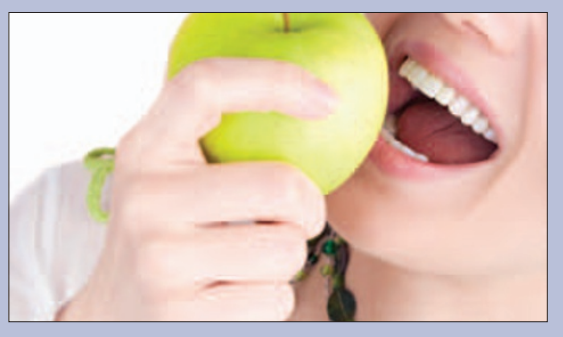

